\title{
Human Milk Oligosaccharides in Colostrum and Mature Milk of Chinese Mothers: Lewis Positive Secretor Subgroups
}

\author{
M. Elwakiel, ${ }^{\dagger, \ddagger}$ J. A. Hageman, ${ }^{\S}$ W. Wang, $"$ I. M. Szeto, $"$ J. B. van Goudoever, ${ }^{\perp}$ K. A. Hettinga, \\ and H. A. Schols* ${ }^{* \dagger}$ \\ ${ }^{\dagger}$ Laboratory of Food Chemistry, and ${ }^{\star}$ Food Quality and Design Group, Wageningen University \& Research, Bornse Weilanden 9, \\ Wageningen $6708 \mathrm{WG}$, The Netherlands \\ ${ }^{\S}$ Biometris-Applied Statistics, Wageningen University \& Research, Droevendaalsesteeg 1, Wageningen 6708 PB, The Netherlands \\ "Inner Mongolia Yili Industrial Group Co., Ltd., Jinshan Road 8, Hohhot 010110, China \\ ${ }^{\perp}$ Department of Pediatrics, Emma Children's Hospital - AMC, Meibergdreef 9, Amsterdam 1100 DD, The Netherlands
}

Supporting Information

ABSTRACT: To study the variability in human milk oligosaccharide (HMO) composition of Chinese human milk over a 20-wk lactation period, HMO profiles of 30 mothers were analyzed using CE-LIF. This study showed that total HMO concentrations in Chinese human milk decreased significantly over a 20-wk lactation period, independent of the mother's SeLe status, although with individual variations. In addition, total acidic and neutral HMO concentrations in Chinese human milk decreased over lactation, and levels are driven by their mother's SeLe status. Analysis showed that total neutral fucosylated HMO concentrations in Chinese human milk were higher in the two secretor groups as compared to the nonsecretor group. On the basis of the total neutral fucosylated HMO concentrations in Chinese human milk, HMO profiles within the Se ${ }^{+} \mathrm{Le}^{+}$ group can be divided into two subgroups. HMOs that differed in level between $\mathrm{Se}^{+} \mathrm{Le}^{+}$subgroups were $2^{\prime} \mathrm{FL}, \mathrm{DF}-\mathrm{L}, \mathrm{LNFP}$, and F-LNO. HMO profiles in Dutch human milk also showed $\mathrm{Se}^{+} \mathrm{Le}^{+}$subgroup division, with $2^{\prime} \mathrm{FL}$, LNT, and F-LNO as the driving force.

KEYWORDS: carbohydrates, variability, lactation stage, genetic polymorphisms

\section{INTRODUCTION}

Human milk is the natural food for infants after birth, providing not only nutrition but also protection against infectious diseases. ${ }^{1}$ Human milk contains a variety of milk components like proteins, lipids, carbohydrates, which support the healthy growth and development of infants. ${ }^{2}$ Specific protective components like oligosaccharides and immune-active proteins in human milk are present in higher concentrations in early lactation than in late lactation, while other nutritional components like lactose and fatty acids increase over lactation. ${ }^{3}$

Lactose and human milk oligosaccharides (HMOs) are both part of the carbohydrate fraction in human milk. ${ }^{4,5}$ Lactose is a disaccharide formed by a $\beta-1,4$ linkage between galactose and glucose, and its concentrations in human milk range from 56 to $69 \mathrm{~g} / \mathrm{L}$ over lactation, ${ }^{3-5}$ although with large individual variation. The enzyme lactase is present in the small intestine, and breaks down lactose into glucose and galactose, ${ }^{6-8}$ although lactose may end up in the colon at early life. HMOs are complex lactose-based glycans synthesized in the mammary gland throughout lactation. ${ }^{9-11}$ HMOs are composed of five monosaccharides: glucose, galactose, $\mathrm{N}$-acetylglucosamine, fucose, and $\mathrm{N}$-acetylneuramic acid. During the synthesis of HMOs, lactose can be elongated by $\beta-1,3$ linkages to lacto- $N$-biose or by $\beta-1,6$ linkages to $N$-acetyllactosamine, and these core $\mathrm{HMO}$ structures can be further decorated with fucose or sialic acid residues. $^{9-11}$ HMOs and lactose are resistant to gastric and duodenal digestion, able to modulate the immune system of the intestinal mucosa, and influence the composition of the gut microbiome. ${ }^{12-17}$ The size, structure, and function differ between HMOs. ${ }^{18}$ More than 100 different structures have been identified and characterized in human milk, including many isomers. ${ }^{19}$ Total HMO concentrations in human milk ranged from 5 to $25 \mathrm{~g} / \mathrm{L}$ over a 6 mo lactation period. ${ }^{19}$ HMOs can be classified as neutral or acidic HMOs, with acidic oligosaccharides generally being present at a 10 -fold lower concentration than neutral oligosaccharides. $^{20,21}$

The type and amount of HMOs present in human milk depend on the genetic profile of the mother, resulting in four major milktypes. $^{22-25}$ Fucosyltransferase (FUT) 2 is encoded by the Se gene and determines the presence of $\alpha 1,2$-fucosylated oligosaccharides in human milk. On the basis of the Lewis blood group system, FUT3 is encoded by the Le gene, which determines the presence of $\alpha 1$,4-fucosylated oligosaccharides in human milk. Women with an active Se locus are classified as secretors $\left(\mathrm{Se}^{+}\right)$, whereas women with an active Le locus are classified as Lewis positive $\left(\mathrm{Le}^{+}\right)$. Women without FUT2 or FUT3 activity are classified as nonsecretors $\left(\mathrm{Se}^{-}\right)$or Lewis negative $\left(\mathrm{Le}^{-}\right)$, lacking $\alpha 1,2$-fucosylated or $\alpha 1$,4-fucosylated oligosaccharides, respectively. A large variation in HMO composition within the four major milk-type groups has been reported, ${ }^{26}$ and might be explained by mutations in the Se and Le genes. ${ }^{27}$ Additional Se

Received: April 18, 2018

Revised: June 15, 2018

Accepted: June 16, 2018

Published: June 16, 2018 


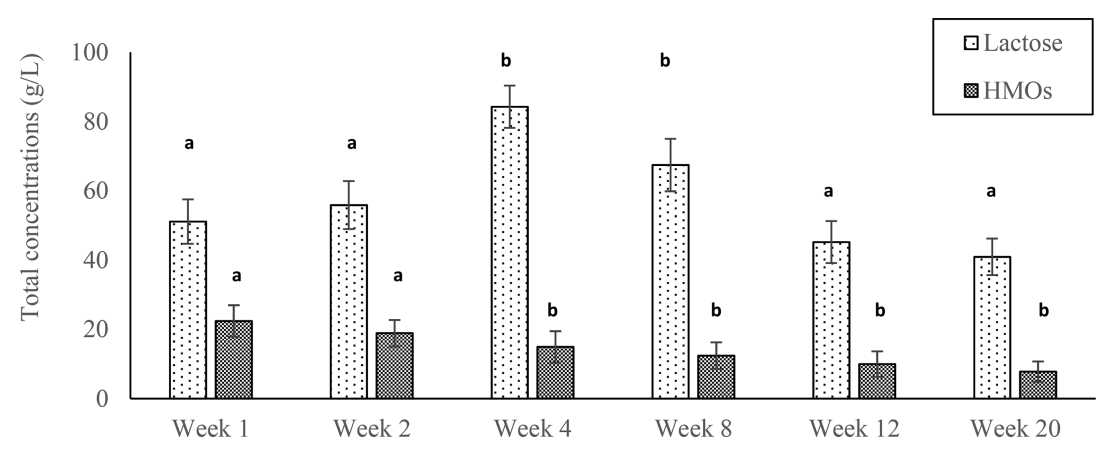

Figure 1. Total lactose and HMO concentrations $(\mathrm{g} / \mathrm{L})$ in Chinese human milk of 30 mothers over a 20-wk lactation period. Error bars indicate the standard deviation. $(\mathrm{a}, \mathrm{b})$ Different alphabet letters indicate different lactose and HMO concentrations in human milk (two-sided $t$-test, $\alpha<0.05)$ between different time points in lactation.

and Le phenotypes have been reported, the so-called weak Se and Le phenotype, respectively, mostly found in the Asian population, ${ }^{27}$ and less common in European population. Weak Se and Le phenotypes are probably able to produce FUT2- and FUT3-mediated oligosaccharides, respectively, with fucosylated HMO levels lower than typically found in regular milk of Se and Le phenotypes. ${ }^{19}$ For example, it has been reported that FUT2- and FUT3-mediated oligosaccharides, such as $2^{\prime}$ fucosyllactose $\left(2^{\prime} \mathrm{FL}\right)$ and $3 \mathrm{FL}$, respectively, can be present in human milk in lower amounts. ${ }^{19} \mathrm{HMO}$ profiles were also shown to be different within and between breastfeeding populations from $>10$ countries. ${ }^{28-30}$ Although human milk of most individuals can be grouped into the four SeLe groups, there exists a large variation in HMO levels within SeLe groups, ${ }^{26,28,31}$ but none of these studies so far tried to find patterns in HMO profiles within the four milk-type groups.

The main objective of this study was to investigate the level and type of HMOs in Chinese human milk over a 20-wk lactation period. HMO profiles of 30 mothers over the course of lactation were investigated using capillary electrophoresis-laserinduced fluorescence (CE-LIF). To investigate whether the observed clustering in HMO composition is typical for only Chinese mothers, HMO profiles of 28 Dutch mothers were determined $4 \mathrm{wk}$ after delivery.

\section{MATERIALS AND METHODS}

Setup of Study and Sample Collection. Chinese participants were recruited between August 2014 and November 2015. The Yili Innovation Center (Hohhot, $\mathrm{CN}$ ) took care of the human milk collection. Women living in the Hohhot region collected milk samples using a human milk pump. For every time point, a volume of $10 \mathrm{~mL}$ was collected in a polypropylene bottle. Milk bottles were shaken gently, aliquoted into $1 \mathrm{~mL}$ Eppendorf tubes, and stored at $-20^{\circ} \mathrm{C}$. Milk samples of 30 mothers were assessed in wk 1, 2, 4, 8, 12, and 20. Human milk collection was approved by the Chinese Ethics Committee of Registering Clinical Trials (ChiECRCT-20150017). Written informed consent was obtained for all of the Chinese participants. Dutch participants were recruited between September 2015 and June 2016. Human milk samples of women who gave birth at the obstetric department of the VU Medical Center in Amsterdam were collected by the Dutch Human Milk Bank. A volume of $10 \mathrm{~mL}$ was collected in a polypropylene bottle and stored at $-20{ }^{\circ} \mathrm{C}$. Milk of 28 Dutch mothers was collected, after 4 wk of delivery. Human milk collection was approved by the VU Medical Center institutional committee, and written informed consent was obtained from all mothers.

Sample Preparation, Labeling, and Data Analysis. HMOs were isolated and extracted from human milk, as described previously. ${ }^{20}$ Defatting of the human milk samples was followed by protein precipitation, and the pellet obtained after centrifugation containing denatured proteins was removed. HMOs present in the supernatant were isolated via solid-phase extraction on graphitized carbon cartridges (Alltech, Deerfield, U.S.). Subsequently, the isolated HMOs were labeled with fluorescent 9-aminopyrene-1,4,6-trisulfonate (APTS), as described previously. ${ }^{20}$ During derivatization, oligosaccharides are linked in a molar ratio of $1: 1$ to the negatively charged label APTS. After labeling of the HMOs, the samples were analyzed using CE-LIF, as described previously. ${ }^{20}$ Samples were measured in triplicate, and xylose was used as the internal standard. HMOs were identified using commercially available standards, and the elution behavior of HMOs was identified in existing literature. ${ }^{20}$ Quantification was done using the molar response factor of APTS labeled xylose, and concentrations compared nicely with known quantities of available HMOs measured. HMO standards $3^{\prime}$ - and $6^{\prime}$-sialyllactose (SL) were bought from Sigma-Aldrich (St. Louis, MO). The HMO standards, $2^{\prime}$ - and 3FL, sialyllacto- $N$-tetraose (S-LNT), LNFP I-III, lacto$N$-difucosylhexaose (LNDFH) I, fucosyllacto- $N$-hexaose (F-LNH) III, and lacto- $N$-hexaose (LNH) were purchased from Dextra (Reading, UK). Difucosyllactose (DF-L) was provided by Elicityl OligoTech (Crolles, FR), while lacto- $N$-tetraose (LNT) and disialyllacto- $N$-tetraose (DS-LNT) were purchased from Carbosynth (Berkshire, UK). For data analysis, Chromeleon 7.1 (Thermo Fisher Scientific, Waltham, U.S.) was used. CE-LIF peak areas were converted to the corresponding HMO concentration in nanomoles $\mathrm{g} / \mathrm{L}$.

Statistical Analysis. Total HMO concentrations in Chinese human milk over lactation were compared and correlated with maternal characteristics (age, parity, body mass index) and socioecomic indicators (employment status and educational background) using SPSS (IBM Corp., NY). The scales for educational background, as well as for employment status, were made from items of a three-point Likert scale. The scale for parity consisted of two. Participants did not have missing values for the categorical items in this study. Distributional aspects of the quantitative variables (age, body mass index, total HMO concentrations) were assessed by histograms (Gaussian distribution), QQ plots (normal distribution), Kolmogorov-Smirnov test (normal distribution), and by asymmetry and kurtosis values (between -3 and 3 ). The values of mother 8 at wk 12 postpartum were excluded from analysis. The quantitative variables were assessed before regression analysis for linearity, univariate and bivariate outliers, and homoscedasticity, using scatterplot matrices, box plots, and residue plots, respectively. For statistical analysis, a $t$-test for independent samples, ANOVA, and multiple linear regression were used. The significance level was set at $\alpha=0.05$.

Human milk was assigned to their mother's SeLe status using 2'FL, LNFP I, LNDFH I, and LNT, as described previously. ${ }^{20}$ The first three structures exclusively qualified the $\mathrm{Se}^{+} \mathrm{Le}^{+}, \mathrm{Se}^{-} \mathrm{Le}^{+}$, and $\mathrm{Se}^{+} \mathrm{Le}^{-}$ groups. In addition, average concentrations of LNT make a clear distinction between group $\mathrm{Se}^{+}$and $\mathrm{Se}^{-}$groups, which can be used as extra information next to the absence of $\alpha 1,2$-fucosylated or $\alpha 1$, 4-fucosylated oligosaccharides in the $\mathrm{Se}^{-} \mathrm{Le}^{-}$group.

Interpretation of the HMO profiles in human milk was facilitated by hierarchical clustering using $\mathrm{R}$ (Lucent Technologies, NJ), with 


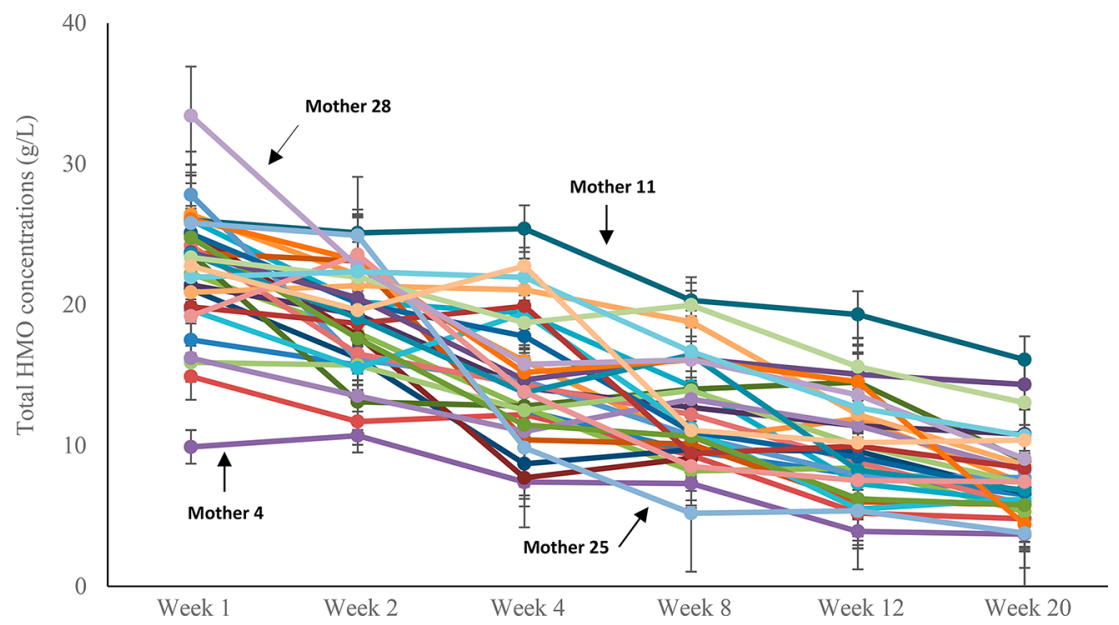

Figure 2. Total HMO concentrations $(\mathrm{g} / \mathrm{L})$ in Chinese human milk of 30 individual mothers over a 20-wk lactation period. Error bars indicate the standard deviation.

Euclidean distance measure and Ward's linkage method. Hierarchical clustering was performed to detect and identify SeLe subgroups based on total, acidic, neutral, and individual HMO concentrations in Chinese human milk over a 20 -wk lactation period. HMO concentrations in Dutch human milk were evaluated in a similar way. The total HMO concentrations are based on 14 HMOs identified in this study, which are expected to present about $90 \%$ of all oligosaccharides present in human milk.

\section{RESULTS AND DISCUSSION}

Total Lactose and HMO Concentrations. To investigate the variability of lactose and HMOs in Chinese human milk over a 20-wk lactation period, lactose and HMO profiles of 30 mothers were analyzed using CE-LIF. Lactose concentrations were ranging from 40 to $85 \mathrm{~g} / \mathrm{L}$ over a 20 -wk lactation period (Figure 1). Lactose levels in Chinese human milk increased in the first $4 \mathrm{wk}$ of lactation, then started to decline. Total HMO concentrations in Chinese human milk, as a sum of all individual HMOs (Supporting Information, data file), were ranging from 8 to $23 \mathrm{~g} / \mathrm{L}$ over lactation (Figure 1). The 14 HMOs identified in this study represent about $90 \%$ of the oligosaccharides present in human milk (Supporting Information, data file). Human milk in early lactation (wk 1 and 2) contained higher total HMO concentrations than in intermediate (wk 4 and 8) and late lactation (wk 12 and 20).

The lactose and total HMO concentrations in Chinese human milk over lactation (Figure 1) match with these observed in earlier studies, with average values of $56-69 \mathrm{~g} / \mathrm{L}^{3}$ and $5-25 \mathrm{~g} / \mathrm{L}^{18}$ respectively, with large individual variation. A change in lactose levels is expected in the first $2 \mathrm{wk}$ of lactation due to the general increase in nutritional components in milk. ${ }^{4,5}$ Transition milk is produced from a couple of days up to $2 \mathrm{wk}$ postpartum, supporting the growth and development of the rapidly growing infant. It has been previously reported that levels of lactose levels are low in colostrum, increase in transitional milk, and then remain constant in mature milk; ${ }^{5}$ however, lactose levels may be more variable in mature milk. ${ }^{4}$ Milk becomes fully mature between 4 and $6 \mathrm{wk}$ postpartum, and contains higher amounts of nutrients as compared to bioactive components. ${ }^{4,5}$ In early life, infants have an immature intestinal immune system, making them more vulnerable to infection by opportunistic pathogens in early lactation. ${ }^{1,2}$ The high HMO level in colostrum may provide protection to the infant in this sensitive stage of its development. ${ }^{10,13}$
Total HMO concentrations in human milk of Chinese mothers over a 20-wk lactation period, as presented in Figure 1, varied significantly among mothers (Figure 2). Although total HMO concentrations were always higher in early lactation than in intermediate and late lactation, the rate of decline varied among mothers. The total HMO concentrations, for example, for mothers 11 and 25 both started around $26 \mathrm{~g} / \mathrm{L}$, although showing the lowest (38\%) and highest (85\%) decline over lactation (Figure 2). As shown in Figure 2, the lowest concentrations in colostrum $(9.9 \mathrm{~g} / \mathrm{L})$ and mature milk $(3.7 \mathrm{~g} / \mathrm{L})$ were linked to mother 4 , whereas the highest concentrations in colostrum $(33.4 \mathrm{~g} / \mathrm{L})$ and mature milk $(25.4 \mathrm{~g} / \mathrm{L})$ were found for mother 28 and 11 , respectively.

Information collected from individual Chinese mothers, their total HMO concentrations, and SeLe status are provided in Table S1. No correlation could be found by ANOVA and multiple regression analysis between the maternal characteristics (age, parity, and socioecomic status) and the total HMO concentrations up to $20 \mathrm{wk}$ (results not shown). Body mass index seems to be positively correlated with total HMO concentrations at wk 1 and 2, whereas no significant relationship was observed at later time points (results not shown). Total HMO concentrations were lower for mothers with a low body mass index in wk 1 and 2. Several studies have suggested that mother's body mass index might influence the total HMO composition in human milk composition, especially colostrum, ${ }^{15,16,28}$ but the underlying mechanism is not yet clear.

Secretor and Lewis Histo-blood Group System. Milk samples were assigned to their mother's SeLe status (Table S1 and Figure S1). Twenty-two out of the 30 Chinese mothers can be assigned to the $\mathrm{Se}^{+} \mathrm{Le}^{+}$group (73\%), while 6 and 2 out of the 30 mothers were assigned to the $\mathrm{Se}^{-} \mathrm{Le}^{+}(20 \%)$ and $\mathrm{Se}^{+} \mathrm{Le}^{-}(7 \%)$ groups, respectively. Milk samples from $\mathrm{Se}^{-} \mathrm{Le}^{-}$ mothers were not present in this study. Distributions of these phenotypes vary among populations, and the frequency of the secretor phenotype in the Chinese population was previously estimated to be between $50 \%$ and $70 \%,{ }^{29,30}$ which match with the findings in this study. The outcomes of this study are also in line with another performed study on Chinese human milk, ${ }^{19}$ where $21 \%$ of the samples contained levels of $2^{\prime} \mathrm{FL}$ below the limit of quantification, similar in frequency for the $\mathrm{Se}^{-} \mathrm{Le}^{+}$group in the European population. ${ }^{19}$ Subsequently, total HMO concentrations in Chinese human milk for the 


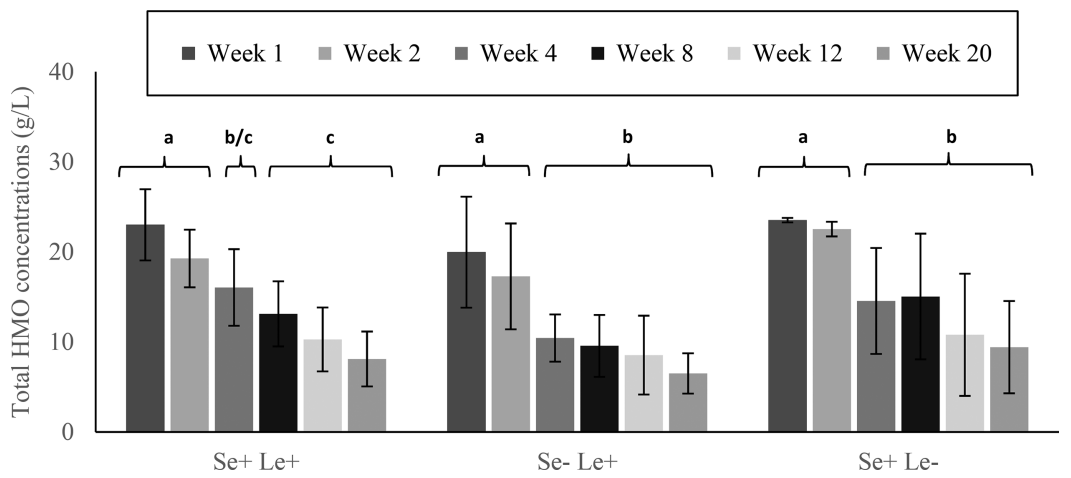

Figure 3. Total HMO concentrations (g/L) in Chinese human milk of 30 mothers over a 20-wk lactation period categorized per SeLe group. Error bars indicate the standard deviation. Se $\mathrm{Le}^{+}$milk-type group $n=22, \mathrm{Se}^{-} \mathrm{Le}^{+}$milk-type group $n=6$, and $\mathrm{Se}^{+} \mathrm{Le}^{-}$milk-type group $n=2$. (a-c) Different alphabet letters indicate different HMO concentrations in human milk (two-sided $t$-test, $\alpha<0.05$ ) between different time points in lactation per SeLe group.

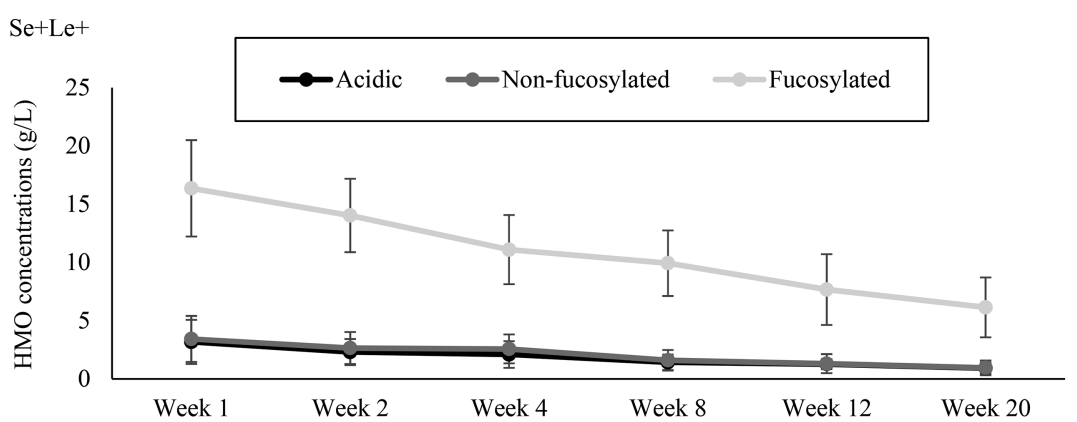

(A)

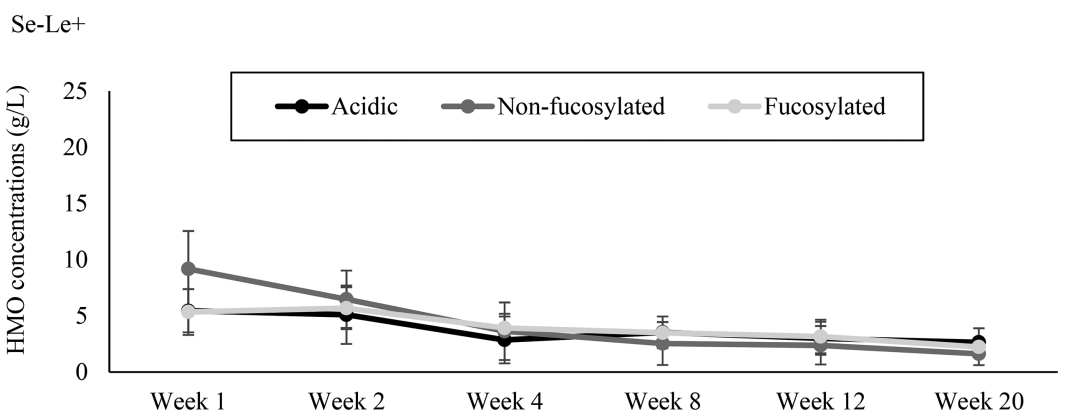

(B)

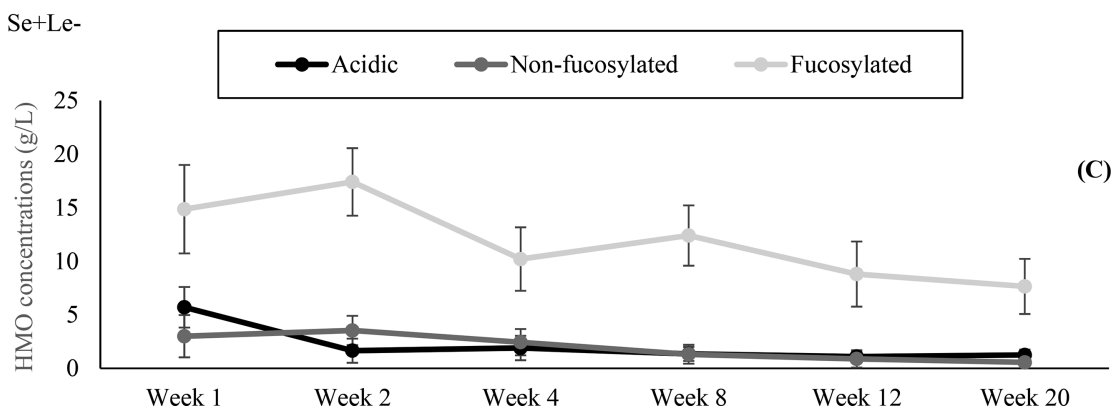

Figure 4. Concentrations of total acidic and total neutral (nonfucosylated and fucosylated) HMO fractions in Chinese human milk over a 20-wk lactation period for (A) Se ${ }^{+} \mathrm{Le}^{+}$milk-type group $n=22$, (B) $\mathrm{Se}^{-} \mathrm{Le}^{+}$milk-type group $n=6$, and (C) $\mathrm{Se}^{+} \mathrm{Le}^{-}$milk-type group $n=2$.

three SeLe groups decreased over a 20-wk lactation period (Figure 3), independent of the mother's SeLe status. The total HMO concentrations in Chinese human milk over a 20-wk lactation period for the $\mathrm{Se}^{+} \mathrm{Le}^{+}, \mathrm{Se}^{-} \mathrm{Le}^{+}$, and $\mathrm{Se}^{+} \mathrm{Le}^{-}$groups were $8.1-23.0,6.5-20.0$, and 9.4-23.5 g/L, respectively, and fall within the range of all combined SeLe groups over lactation. $^{18}$
Acidic and Neutral HMO Concentrations. The total acidic and total neutral HMO fractions in human milk per mother and per time point in lactation are available in Table S2. For both the $\mathrm{Se}^{+} \mathrm{Le}^{+}(n=22)$ and the $\mathrm{Se}^{+} \mathrm{Le}^{-}(n=2)$ groups (Figure 4, $\mathrm{A}$ and $\mathrm{C}$ ), the concentrations for the total neutral fucosylated HMO fraction decreased with 10.3 and $7.2 \mathrm{~g} / \mathrm{L}$ over lactation, respectively, while the concentrations of the total acidic and 
neutral nonfucosylated HMO fractions even decreased relatively faster over lactation. For the $\mathrm{Se}^{-} \mathrm{Le}^{+}(n=6)$ milk type, the concentrations for the total neutral nonfucosylated HMO fraction were decreasing the most with $7.6 \mathrm{~g} / \mathrm{L}$ over lactation (Figure $4 \mathrm{~B}$ ).

For the $\mathrm{Se}^{+}$groups, higher amounts were found for the total neutral fucosylated $\mathrm{HMO}$ fraction as compared to the $\mathrm{Se}^{-}$ group. Despite the absence of the FUT2 enzyme for the $\mathrm{Se}^{-} \mathrm{Le}^{+}$group, and different profiles of three groups of HMOs in Chinese human milk over a 20 -wk lactation period, concentrations of the total neutral nonfucosylated HMO fraction might function as compensation, which possibly explains why the total HMO concentration ends up being the same for all genetic groups (Figure 3). However, having very few individuals in the $\mathrm{Se}^{-} \mathrm{Le}^{+}$and $\mathrm{Se}^{+} \mathrm{Le}^{-}$milk-type groups complicates comparison between groups. The concentrations of the three groups of HMOs expressed in percentages in Chinese human milk for the $\mathrm{Se}^{+} \mathrm{Le}^{+}$and $\mathrm{Se}^{-} \mathrm{Le}^{+}$milk-type groups over lactation can be found in Figure S2. The data of the $\mathrm{Se}^{+} \mathrm{Le}^{-}$milk-type group are not displayed in Figure S2, because it showed identical patterns over time with the $\mathrm{Se}^{+} \mathrm{Le}^{+}$milk-type. The ratios between total acidic and total neutral HMO concentrations for the $\mathrm{Se}^{+} \mathrm{Le}^{+}$milk-type and $\mathrm{Se}^{-} \mathrm{Le}^{+}$milk-type group were ranging from $13: 87$ to $12: 88$ and from $28: 72$ to $40: 60$ over lactation (Figure S2), respectively, indicating that acidic HMOs over time might be relatively more dominant in the $\mathrm{Se}^{-} \mathrm{Le}^{+}$milktype group than in the $\mathrm{Se}^{+} \mathrm{Le}^{+}$milk-type group. Overall, total acidic and total neutral HMO concentrations in Chinese human milk per SeLe group vary over the course of lactation, with overall higher total neutral HMO concentration in all groups.

$\mathrm{Se}^{+} \mathrm{Le}^{+}$Subgroups in Chinese Human Milk. To investigate the observed variability in total acidic and total neutral (nonfucosylated and fucosylated) HMO concentrations in human milk of Chinese mothers for the three SeLe groups over a 20-wk lactation period, total acidic and total neutral HMO concentrations per mother were examined by clustering analysis. Statistical analysis confirmed the clear difference that exists between $\mathrm{Se}^{-}$and $\mathrm{Se}^{+}$groups (Figure 5, cluster I/II versus III). However, with concentrations of the total acidic and total neutral (nonfucosylated and fucosylated) HMO fractions in human milk per mother, $\mathrm{Se}^{+} \mathrm{Le}^{+}$mothers could be divided into two subgroups (Figure 5, clusters I and II). The size of the $\mathrm{Se}^{-} \mathrm{Le}^{+}(n=6)$ and $\mathrm{Se}^{+} \mathrm{Le}^{-}(n=2)$ groups was too small to detect any subgroups. Cluster III consisted only of $\mathrm{Se}^{-} \mathrm{Le}^{+}$mothers. Milk from the two mothers having $\mathrm{Se}^{+} \mathrm{Le}^{-}$could not be clustered and end up in the $\mathrm{Se}^{+} \mathrm{Le}^{+}$group (Figure 5).

The $2 \mathrm{Se}^{+} \mathrm{Le}^{+}$subgroups, displayed in Figure 5, seem to be distinguished by their total neutral fucosylated HMO fraction (Figure 6). The concentrations of the total neutral fucosylated HMO fraction are significantly different and are almost $20 \%$ higher in subgroup I than in subgroup II (Figure 6). The concentrations of the total acid and total neutral nonfucosylated HMO fraction did not differ significantly between the $2 \mathrm{Se}^{+} \mathrm{Le}^{+}$subgroups.

The phenomena of the $\mathrm{Se}^{+} \mathrm{Le}^{+}$subgroup formation might be a consequence of the observation that Se and Le genes can contain mutations. ${ }^{27}$ Besides the full absence of FUT2 and FUT3, two different phenotypes have been found, so-called weak Se and Le phenotypes, respectively. ${ }^{27}$ Because of modifications in the amino acid sequence, the activity of the FUT2 or FUT3 enzyme can be reduced, thereby possibly leading to a decrease in the synthesis of HMOs in one of the subgroups. ${ }^{19}$ From the table containing all individual HMO concentrations (Supporting Information, data file), it could be deducted that HMOs that

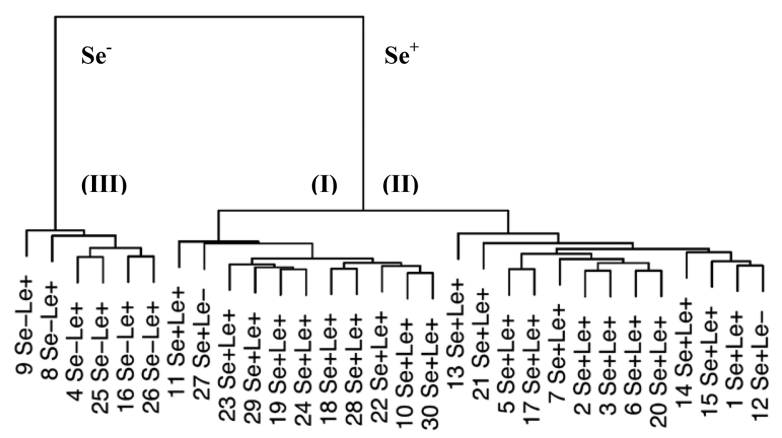

Figure 5. Hierarchical cluster analysis of total acidic and total neutral (nonfucosylated and fucosylated) HMO concentrations $(\mathrm{g} / \mathrm{L})$ in Chinese human milk per mother over a 20 -wk lactation period. (I) $\mathrm{Se}^{+} \mathrm{Le}^{+}$milktype group I, (II) $\mathrm{Se}^{+} \mathrm{Le}^{+}$milk-type group II, and (III) $\mathrm{Se}^{-} \mathrm{Le}^{+}$milk-type.

differed between $\mathrm{Se}^{+} \mathrm{Le}^{+}$subgroups were $2^{\prime} \mathrm{FL}$, DF-L, LNFP I, and F-LNO (Figure 7), having in common $\alpha 1,2$-fucosylated linkages to the core HMO structures.

The reason for the variation in these specific HMOs in this study (Figure 7) is not yet clarified. However, previous studies have reported that levels of 2'FL and LNFP I were found below normal ranges in human milk from a small group of Chinese participants. ${ }^{19}$ Gene mutations are not limited to the FUT2 enzyme activity, because various mutations have also been reported in the Le gene encoding for the FUT3 enzyme. In human milk collected from the Chinese mothers, variation based on FUT3-mediated oligosaccharides in $\mathrm{Se}^{+} \mathrm{Le}^{+}$and $\mathrm{Se}^{-} \mathrm{Le}^{+}$groups could not be seen. Additionally, it has been noticed that 3FL, as indicator for the FUT3 enzyme, was removed in the pretreatment step, and therefore 3FL was not able to be detected in the samples of this study.

$\mathrm{Se}^{+} \mathrm{Le}^{+}$Subgroups in Dutch Human Milk. To investigate whether the observed differentiation in $\mathrm{Se}^{+} \mathrm{Le}^{+}$subgroups in Chinese human milk also applies to other populations, HMO profiles from 28 Dutch mothers were collected 4 wk after delivery and analyzed. Total HMO concentrations measured in Dutch human milk, as a sum of the $14 \mathrm{HMOs}$ (Supporting Information, data file), ranged from 4 to $27 \mathrm{~g} / \mathrm{L} 4 \mathrm{wk}$ postpartum (Table S3), independent of the mother's SeLe status and body mass index (results not shown).

Milk samples were also assigned to their mother's SeLe status (Table 3). Fourteen Dutch mothers can be assigned to the $\mathrm{Se}^{+} \mathrm{Le}^{+}$group (50\%), while 11 and 3 mothers are identified as belonging to the $\mathrm{Se}^{-} \mathrm{Le}^{+}(39 \%)$ and $\mathrm{Se}^{+} \mathrm{Le}^{-}$(11\%) groups, respectively. Milk samples from $\mathrm{Se}^{-} \mathrm{Le}^{-}$mothers were not present in this study. The distribution over the four SeLe groups for the Dutch mothers did not correspond with previously reported numbers reporting 70-80\% being $\mathrm{Se}^{+} \mathrm{Le}^{+}$for the European population $^{21-25}$ and $80 \%$ for the Dutch population. ${ }^{20}$ These unexpected proportions should not affect the analysis, as the group for $\mathrm{Se}^{+} \mathrm{Le}^{+}$mothers was large enough to perform cluster analysis, and the observed uncommon ratio between SeLe groups made it even possible to investigate the $\mathrm{Se}^{-} \mathrm{Le}^{+}$group in more detail. After clustering analysis, $\mathrm{HMO}$ levels in milk of 28 Dutch mothers also showed $\mathrm{Se}^{+} \mathrm{Le}^{+}$ subgroup division (Figure 8). Two $\mathrm{Se}^{+} \mathrm{Le}^{+}$mothers (8\%) did not fall in either the $\mathrm{Se}^{+} \mathrm{Le}^{+}$subgroup I or II (Figure 8). The $\mathrm{Se}^{-} \mathrm{Le}^{+}$milk-type $(39 \%)$ can be roughly divided into group $\mathrm{III}=$ $6(21 \%)$, group IV $=2(11 \%)$, and group $\mathrm{V}=3(7 \%)$.

As shown in Figure 8, there is a lot of variation in $\mathrm{HMO}$ concentrations in Dutch human milk for the $\mathrm{Se}^{-} \mathrm{Le}^{+}$group (cluster III-V). However, no significant difference could be 


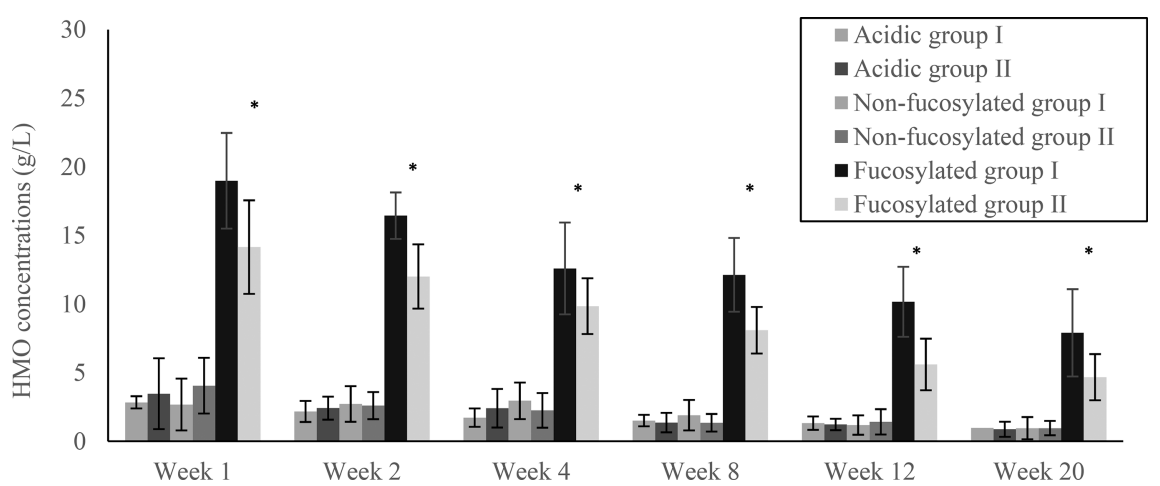

Figure 6. Concentrations of the total acidic and total neutral (fucosylated and nonfucosylated) HMO fractions for the two $\mathrm{Se}^{+} \mathrm{Le}^{+}$subgroups in Chinese human milk over a 20 -wk lactation period. The $\mathrm{Se}^{+} \mathrm{Le}^{+}$milk-type group ( 22 of the 30 mothers, $73 \%$ ) can be divided into group I = 12 $(40 \%)$ and group II $=10(33 \%)$. “*” indicates significant differences (two-sided $t$-test, $\alpha<0.05)$.

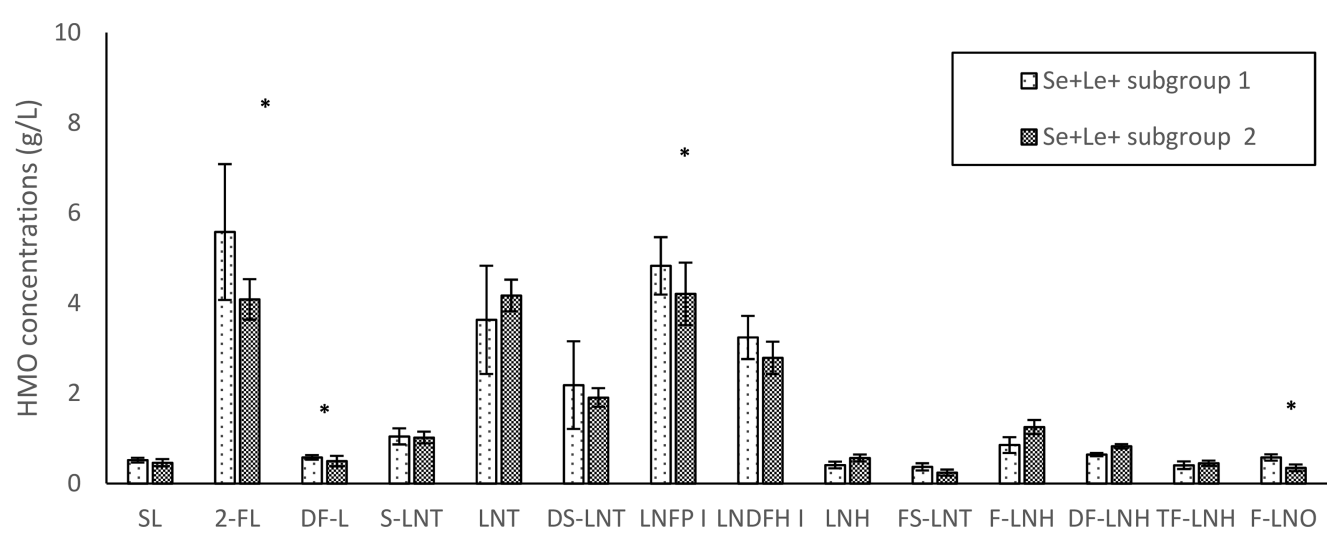

Figure 7. HMO concentrations $(\mathrm{g} / \mathrm{L})$ in Chinese human milk over a 20 -wk lactation period per $\mathrm{Se}^{+} \mathrm{Le}^{+}$subgroup. (I) $\mathrm{Se}^{+} \mathrm{Le}^{+}$milk-type group I, (II) $\mathrm{Se}^{+} \mathrm{Le}^{+}$milk-type group II. “*” indicates significant differences (two-sided $t$-test, $\alpha<0.05$ ). Other than $2^{\prime} \mathrm{FL}$, LNFP I, and LNDFH I, isomers are not further specified.

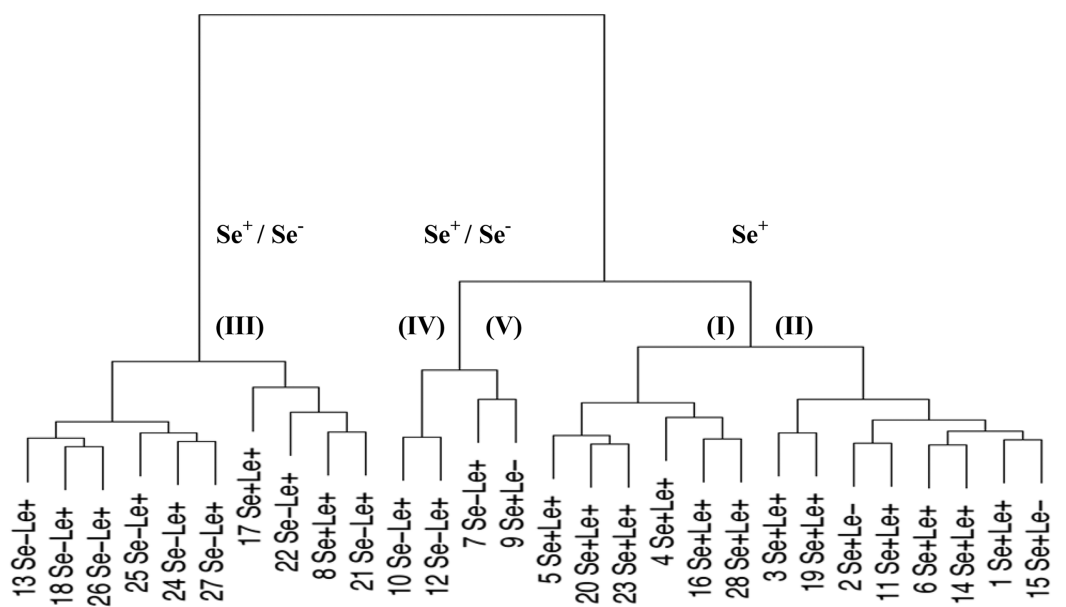

Figure 8. Hierarchical clustering analysis based on concentrations of the total acidic and total neutral (nonfucosylated and fucosylated) HMO fractions $(\mathrm{g} / \mathrm{L})$ in Dutch human milk per mother collected after 4 wk of delivery.

found in concentrations of the total acidic and total neutral (nonfucosylated and fucosylated) HMO fractions between the $\mathrm{Se}^{-} \mathrm{Le}^{+}$subgroups (data not shown). Milk of the Dutch mothers categorized in the $\mathrm{Se}^{+} \mathrm{Le}^{+}$group (Figure 8) can be divided into two subgroups (I and II) on the basis of the concentrations of the neutral fucosylated HMO fraction (Figure 9), like it was done for the $\mathrm{Se}^{+} \mathrm{Le}^{+}$group in Chinese human milk (Figure 6), however, with $2^{\prime} \mathrm{FL}, \mathrm{LNT}$, and F-LNO contributing to the differentiation more than the other HMOs (Figure 10).
Although the levels of HMOs, like DF-L and LNFP I, do not differ significantly between the $\mathrm{Se}^{+} \mathrm{Le}^{+}$subgroups in Dutch human milk (Figure 10), a trend was visible that concentrations were slightly higher for $\mathrm{Se}^{+} \mathrm{Le}^{+}$subgroup I as compared to subgroup II, which was much more clear for Chinese human milk. Levels of DF-L and LNFP I were significantly different between the $\mathrm{Se}^{+} \mathrm{Le}^{+}$subgroups in Chinese human milk, also higher in $\mathrm{Se}^{+} \mathrm{Le}^{+}$subgroup I than in subgroup II (Figure 7). Subsequently, concentrations for LNT in Chinese human milk 


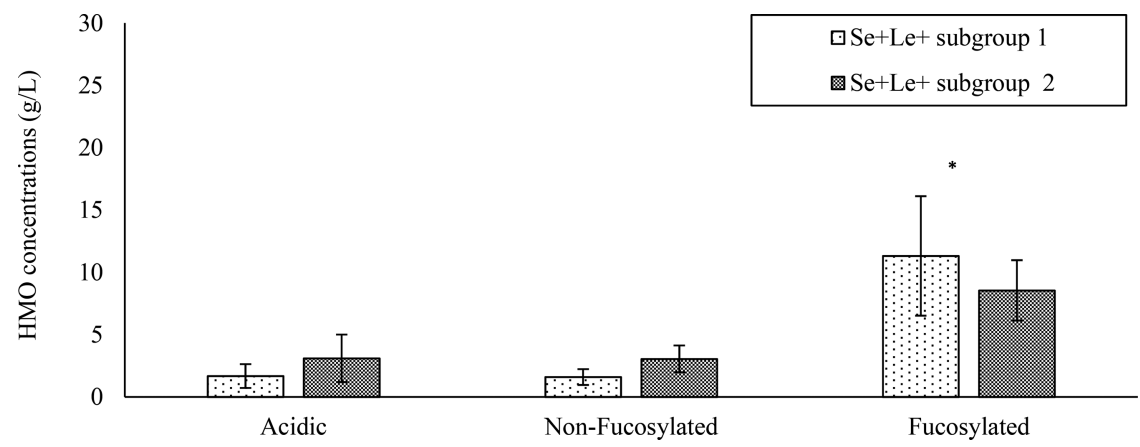

Figure 9. Concentrations of the total acidic and total neutral (fucosylated and nonfucosylated) HMO fractions for the two $\mathrm{Se}^{+} \mathrm{Le} \mathrm{e}^{+}$subgroups in Dutch human milk collected 4 wk postpartum. The $\mathrm{Se}^{+} \mathrm{Le}^{+}$milk-type group (14 of the 28 mothers, 50\%) can be divided into group $\mathrm{I}=6(21 \%)$, group II $=6(21 \%)$, and group IV $=2(8 \%)$. The two $\mathrm{Se}^{+} \mathrm{Le}^{+}$mothers $(8 \%)$, which could not be grouped into $\mathrm{Se}^{+} \mathrm{Le}^{+}$subgroup I and II, were excluded from comparison. "*” indicates significant differences (two-sided $t$-test, $\alpha<0.05$ ).

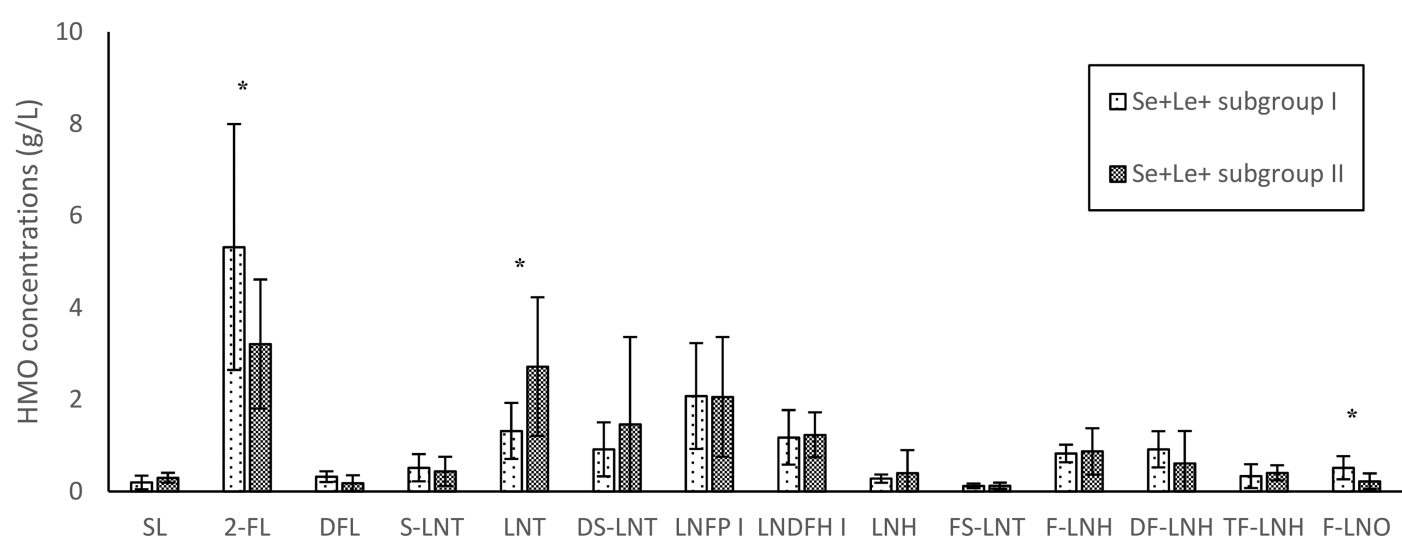

Figure 10. HMO concentrations (g/L) of the two $\mathrm{Se}^{+} \mathrm{Le}^{+}$subgroups in Dutch human milk after 4 wk of delivery. (I) Se ${ }^{+} \mathrm{Le}^{+}$milk-type group I, (II) $\mathrm{Se}^{+} \mathrm{Le}^{+}$milk-type group II. “*” indicates significant differences (two-sided $t$-test, $\alpha<0.05$ ). Other than $2^{\prime} \mathrm{FL}$, LNFP I, and LNDFH I, isomers are not further specified.

were significantly higher for $\mathrm{Se}^{+} \mathrm{Le}^{+}$subgroup II than subgroup I (Figure 7); such a trend could also be observed in Dutch human milk (Figure 10), although not significantly different. Overall, FUT2-mediated HMO structures play a key role in the differentiation between the subgroups in both Chinese and Dutch human milk, indicating that enzyme activity may be reduced for the FUT2 enzyme due to polymorphism.

This study tried to fill a gap in the literature by trying to recognize subgroups with statistics and highlight the variability in HMO composition in Chinese human milk of 30 mothers over a 20-wk lactation period. This study showed that total HMO concentrations in Chinese human milk are not driven by their mother's SeLe status, but ratios of the total acidic and total neutral HMO fractions in human milk of Chinese mothers are responsible for the clustering. On the basis of the neutral fucosylated $\mathrm{HMO}$ fraction, $\mathrm{Se}^{+} \mathrm{Le}^{+}$subgroups were recognized. To investigate whether the observed variability in $\mathrm{HMO}$ composition is typical for only Chinese mothers, HMO profiles of 28 Dutch mothers $4 \mathrm{wk}$ postpartum were investigated, which resulted in $\mathrm{Se}^{+} \mathrm{Le}^{+}$subgroups, based on the concentrations of the neutral fucosylated HMO fraction, although with distinctive HMOs having a different concentration for the two subgroups.

\section{ASSOCIATED CONTENT}

\section{S Supporting Information}

The Supporting Information is available free of charge on the ACS Publications website at DOI: 10.1021/acs.jafc.8b02021.
Raw data of individual HMO concentrations in Chinese and Dutch human milk per mother (PDF)

Tables S1-S3 and Figures S1 and S2 (PDF)

\section{AUTHOR INFORMATION}

\section{Corresponding Author}

*Tel.: +31 317 482239. E-mail: henk.schols@wur.nl. ORCID

H. A. Schols: 0000-0002-5712-1554

Funding

This study has been financially supported by Inner Mongolia Yili Industrial Group Co., Ltd.

Notes

The authors declare no competing financial interest.

\section{ACKNOWLEDGMENTS}

We want to thank Fang Fang (Yili Innovation Center, Inner Mongolia Yili Industrial Group Co., Ltd., Hohhot, China) and Marita de Waard (Department of Paedictrics, VU Medical Center, Amsterdam, The Netherlands) for collecting the Chinese and Dutch human milk, respectively.

\section{ABBREVIATIONS USED}

APTS, 9-aminopyrene-1,4,6-trisulfonate; CE-LIF, capillary electrophoresis laser-induced fluorescence; DF-L, difucosyllactose; DF-LNH, difucosyllacto- $N$-hexaose; DS-LNT, disialyllacto- $N$ tetraose; FL, fucosyllactose; F-LNH, fucosyllacto- $N$-hexaose; 
FS-LNT, fucosyl-sialyllacto- $N$-tetraose; F-LNO, fucosyllacto$\mathrm{N}$-octaose; FUT, fucosyltransferase; HMO, human milk oligosaccharide; HMOs, human milk oligosaccharides; LNDFH, lacto- $N$-difucosylhexaose; LNFP, lacto- $N$-fucopentaose; LNH, lacto- $N$-hexaose; LNT, lacto- $N$-tetraose; $\mathrm{Se}^{+} \mathrm{Le}^{+}$, secretor Lewis positive; $\mathrm{Se}^{-} \mathrm{Le}^{+}$, nonsecretor Lewis positive; $\mathrm{Se}^{+} \mathrm{Le}^{-}$, secretor Lewis negative; $\mathrm{Se}^{-} \mathrm{Le}^{-}$, nonsecretor Lewis negative; SL, sialyllactose; S-LNT, sialyllacto- $N$-tetraose; TF-LNH, trifucosyllacto- $N$-hexaose

\section{REFERENCES}

(1) Leung, A.; Sauve, R. Breast is best for babies. J. Natl. Med. Assoc. 2005, 97, 1010-9.

(2) Labbok, M.; Clark, D.; Goldman, A. Breastfeeding: Maintaining an irreplaceable immunological resource. Nat. Rev. Immunol. 2004, 4, 565-72.

(3) Coppa, G.; Gabrielli, O.; Pierani, P.; Catassi, C.; Carlucci, A.; Giorgi, P. Changes in carbohydrate composition in human milk over 4 months of lactation. Pediatrics 1993, 91, 637-41.

(4) Newburg, D. Glycobiology of human milk. Biochemistry 2013, $78,771-85$.

(5) Ballard, O.; Morrow, A. Human milk composition: Nutrients and bioactive factors. Pediatr. Clin. North Am. 2013, 60, 49-74.

(6) Schaafsma, G. Lactose and lactose derivatives as bioactive ingredients in human nutrition. Int. Dairy J. 2008, 18, 458-65.

(7) Tran, H.; Moreno, R.; Hinkle, E.; Bundy, J.; Walter, J.; Burkey, T.; Miller, P. Effect of lactose and yeast-dried milk on growth performance, fecal microbiota, and immune parameters of nursery pigs. J. Anim. Sci. 2012, 90, 3049-59.

(8) Cederlund, A.; Kai-Larsen, Y.; Printz, G.; Yoshio, H.; Alvelius, G.; Lagercrantz, H.; Strömberg, R.; Jörnvall, H.; Gudmundsson, G.; Agerberth, B. Lactose in human breast milk an inducer of innate immunity with implications for a role in intestinal homeostasis. PLoS One 2013, 8, 1-12.

(9) Stahl, B.; Thurl, S.; Zeng, J.; Karas, M.; Hillenkamp, F.; Steup, M.; Sawatzki, G. Oligosaccharides from human milk as revealed by matrix-assisted laser desorption/ionization mass spectrometry. Anal. Biochem. 1994, 223, 218-26.

(10) Kunz, C.; Rudloff, S.; Baier, W.; Klein, N.; Strobel, S. Oligosaccharides in human milk: Structural, functional, and metabolic aspects. Annu. Rev. Nutr. 2000, 20, 699-722.

(11) Boehm, G.; Stahl, B. Oligosaccharides from milk. J. Nutr. 2007, $137,847-9$

(12) Newburg, D. Oligosaccharides in human milk and bacterial colonization. J. Pediatr. Gastroenterol. Nutr. 1999, 30, 8-17.

(13) Kunz, C.; Rudloff, S. Health promoting aspects of milk oligosaccharides. Int. Dairy J. 2006, 16, 1341-6.

(14) Bode, L.; Jantscher-Krenn, E. Structure-function relationships of human milk oligosaccharides. Adv. Nutr. 2012, 3, 383-91.

(15) Lewis, Z.; Totten, S.; Smilowitz, J.; Popoviv, M.; Parker, E.; Lemay, D.; Van Tassell, M.; Miller, M.; Jin, Y.; German, B.; Lebrilla, C.; Mills, D. Maternal fucosyltransferase 2 status affects the gut bifidobacterial communities of breastfed infants. Microbiome 2015, 11, $1-21$.

(16) Sprenger, N.; Odenwald, H.; Kukkonen, A. K.; Kuitunen, M.; Savilahti, E.; Kunz, C. FUT2-dependent breast milk oligosaccharides and allergy at 2 and 5 years of age in infants with high hereditary allergy risk. Eur. J. Nutr. 2017, 56, 1293-1301.

(17) Seppo, A.; Austran, C.; Bode, L.; Järvinen, K. Human milk oligosaccharides and development of cow's milk allergy in infants. $J$. Allergy Clin. Immunol. 2017, 139, 708-11.

(18) Bode, L. Human milk oligosaccharides: Every baby needs a sugar mama. Glycobiology 2012, 22, 1147-62.

(19) Austin, S.; De Castro, C.; Benet, T.; Hou, Y.; Sun, H.; Thakkar, S.; Vinyes-Pares, G.; Zhang, Y.; Wang, P. Temporal change of the content of 10 oligosaccharides in the milk of Chinese urban mothers. Nutrients 2016, 8, 346-68.
(20) Albrecht, S.; Schols, H.; van den Heuvel, E.; Voragen, A. Occurrence of oligosaccharides in feces of breast-fed babies in their first six months of life and the corresponding breast milk. Carbohydr. Res. 2011, 346, 2540-50.

(21) Thurl, S.; Munzert, M.; Henker, J.; Boehm, G.; Muller-Werner, B.; Jelinek, J.; Stahl, B. Variation of human milk oligosaccharides in relation to milk groups and lactational periods. Br. J. Nutr. 2010, 104, 1261-71.

(22) Grollman, E.; Kobata, A.; Ginsburg, V. An enzymatic basis for Lewis blood types in man. J. Clin. Invest. 1969, 48, 1489-94.

(23) Thurl, S.; Henker, J.; Siegel, M.; Tovar, K.; Sawatzki, G. Detection of four human milk groups with respect to Lewis blood group dependent oligosaccharides. Glycoconjugate J. 1997, 14, 795-9.

(24) Newburg, D. Are all human milks created equal? Variation in human milk oligosaccharides. J. Pediatr. Gastroenterol. Nutr. 2000, 30, $131-3$.

(25) Blank, D.; Dotz, V.; Geyer, R.; Kunz, C. Human milk oligosaccharides and Lewis blood group: Individual high-throughput sample profiling to enhance conclusions from functional studies. $A d v$. Nutr. 2012, 3, 440-9.

(26) Prieto, P. Profiles of human milk oligosaccharides and production of some human milk oligosaccharides in transgenic animals. Adv. Nutr. 2012, 3, 456-64.

(27) Schenkel-Brunner, H. Human Blood Groups: Chemical and Biochemical Basis of Antigen Specificity; Springer: Vienna, AT, 2000; pp 205-212.

(28) McGuire, M. What's normal? Oligosaccharide concentrations and profiles in milk produced by healthy women vary geographically. Am. J. Clin. Nutr. 2017, 105, 1086-100.

(29) Erney, R.; Malone, W.; Skelding, M.; Marcon, A.; KlemanLeyer, K.; O’Ryan, M.; Ruiz-Palacios, G.; Hilty, M.; Pickering, L.; Prieto, P. Variability of human milk neutral oligosaccharides in a diverse population. J. Pediatr. Gastroenterol. Nutr. 2000, 30, 181-92.

(30) Castanys-Muoz, E.; Martin, M.; Prieto, P. 2'fucosyllactose: An abundant, genetically determined soluble glycan present in human milk. Nutr. Rev. 2013, 71, 773-89.

(31) Sprenger, N.; Lee, L.; De Castro, C.; Steenhout, P.; Thakkar, K. Longitudinal change of selected human milk oligosaccharides and association to infants' growth, an observatory, single center, longitudinal cohort study. PLoS One 2017, 12, 1-15. 